1. Ransohoff, R.M. 2006. EAE: pitfalls outweigh virtues of screening potential treatments for multiple sclerosis. Trends Immunol. 27:167-168.

2. Krishnamoorthy, G., Lassmann, H., Wekerle, H., and Holz, A. 2006. Spontaneous opticospinal encephalomyelitis in a double-transgenic mouse model of autoimmune $\mathrm{T}$ cell/B cell cooperation. J. Clin. Invest. 116:2385-2392. doi:10.1172/JCI28330.

3. Bettelli, E., Baeten, D., Jäger, A., Sobel, R.A., and Kuchroo, V.K. 2006. Myelin oligodendrocyte glycoprotein-specific $\mathrm{T}$ and $\mathrm{B}$ cells cooperate to induce a Devic-like disease in mice. J. Clin. Invest. 116:2393-2402. doi:10.1172/JCI28334.

4. Litzenburger, T., et al. 1998. B lymphocytes producing demyelinating autoantibodies: development and function in gene-targeted transgenic mice. J. Exp. Med. 188:169-180.

5. Bettelli, E., et al. 2003. Myelin oligodendrocyte glycoprotein-specific $\mathrm{T}$ cell receptor transgenic mice develop spontaneous optic neuritis. J. Exp. Med. 197:1073-1081.

6. Storch, M.K., et al. 1998. Autoimmunity to myelin oligodendrocyte glycoprotein in rats mimics the spectrum of multiple sclerosis pathology. Brain Pathol. 8:681-694.

7. Wekerle, H., and Linington, C. 2006. Organ specific autoantigens and the autoreactive $T$ cell repertoire: the case of myelin oligodendrocyte glycoprotein. Eur. J. Immunol. 36:512-515.

8. Fazilleau, N., et al. 2006. Persistence of autoreactive myelin oligodendrocyte glycoprotein (MOG)specific T cell repertoires in MOG-expressing mice. Eur. J. Immunol. 36:533-543.

9. Kroepfl, J.F., Viise, L.R., Charron, A.J., Linington, C., and Gardinier, M.V. 1996. Investigation of myelin/oligodendrocyte glycoprotein membrane topology. J. Neurochem. 67:2219-2222.

10. Mathey, E., Breithaupt, C., Schubart, A.S., and Linington, C. 2004. Commentary: Sorting the wheat from the chaff: identifying demyelinating components of the myelin oligodendrocyte glycoprotein (MOG)-specific autoantibody repertoire. Eur. J. Immunol. 34:2065-2071.

11. Madsen, L.S., et al. 1999. A humanized model for multiple sclerosis using HLA-DR2 and a human T-cell receptor. Nat. Genet. 23:343-347.

12. Goverman, J. 1999. Tolerance and autoimmunity in TCR transgenic mice specific for myelin basic protein. Immunol. Rev. 169:147-159.

13. Furtado, G.C., et al. 2001. Regulatory T cells in spontaneous autoimmune encephalomyelitis. Immunol. Rev. 182:122-134.

14. Waldner, H., Whitters, M.J., Sobel, R.A., Collins, M. and Kuchroo, V.K. 2000. Fulminant spontaneous autoimmunity of the central nervous system in mice transgenic for the myelin proteolipid proteinspecific T cell receptor. Proc. Natl. Acad. Sci. U. S. A. 97:3412-3417.

15. Westland, K.W. et al. 1999. Activated non-neura specific $\mathrm{T}$ cells open the blood-brain barrier to circulating antibodies. Brain. 122:1283-1291.

16. Rodriguez-Pinto, D. 2005. B cells as antigen presenting cells. Cell. Immunol. 238:67-75.

17. Matthys, P., Vermeire, K., Heremans, H., and Billiau, A. 2000. The protective effect of IFN-gamma in experimental autoimmune diseases: a central role of mycobacterial adjuvant-induced myelopoiesis. J. Leukoc. Biol. 68:447-454.

18. Kawakami, N., et al. 2004. The activation status of neuroantigen-specific $\mathrm{T}$ cells in the target organ determines the clinical outcome of autoimmune encephalomyelitis. J. Exp. Med. 199:185-197.
19. Linington, C., et al. 1993. T cells specific for the myelin oligodendrocyte glycoprotein mediate an unusual autoimmune inflammatory response in the central nervous system. Eur. J. Immunol. 23:1364-1372.

20. Berger, T., et al. 1997. Experimental autoimmune encephalomyelitis: the antigen specificity of $\mathrm{T}$ lymphocytes determines the topography of lesions in the central and peripheral nervous system. Lab. Invest. 76:355-364.

21. Stadelmann, C., and Bruck, W. 2004. Lessons from the neuropathology of atypical forms of multiple sclerosis. Neurol. Sci. 25(Suppl. 4):S319-S322.

22. Hughes, R.A., and Cornblath, D.R. 2005. GuillainBarre syndrome. Lancet. 366:1653-1666.

23. Willison, H.J. 2006. Basic and clinical aspects of autoimmune disorders in peripheral nerves. Acta Neurol. Scand. Suppl. 183:14-18.

24. Cree, B.A., Goodin, D.S., and Hauser, S.L. 2002. Neuromyelitis optica. Semin. Neurol. 22:105-122.

25. Giovannoni, G. 2006. Neuromyelitis optica and anti-aquaporin-4 antibodies: widening the clinical phenotype. J. Neurol. Neurosurg. Psychiatry. doi:10.1136/jnnp.2006.090944

26. Lucchinetti, C.F., et al. 2002. A role for humoral mechanisms in the pathogenesis of Devic's neuromyelitis optica. Brain. 125:1450-1461.

27. Wingerchuk, D.M. 2006. Neuromyelitis optica. Int. MS J. 13:42-50.

28. Lennon, V.A., et al. 2004. A serum autoantibody marker of neuromyelitis optica: distinction from multiple sclerosis. Lancet. 364:2106-2112.

29. Lennon, V.A., Kryzer, T.J., Pittock, S.J., Verkman, A.S., and Hinson, S.R. 2005. IgG marker of opticspinal multiple sclerosis binds to the aquaporin-4 water channel. J. Exp. Med. 202:473-477.

\title{
Regeneration of the endothelium as a novel therapeutic strategy for acute lung injury
}

\author{
Tohru Minamino and Issei Komuro
}

Department of Cardiovascular Science and Medicine, Chiba University Graduate School of Medicine, Chiba, Japan.

\begin{abstract}
Acute lung injury (ALI) is characterized by the influx of protein-rich edematous fluid into the airspaces due to increased permeability of the alveolarcapillary barrier. Inflammatory mediators are thought to play a critical role in the pathogenesis of this disorder. In this issue of the JCI, Zhao et al. report that the forkhead box M1 (FoxM1) transcription factor induces endothelial regeneration and thereby restores endothelial barrier function after ALI (see the related article beginning on page 2333). Their findings raise the intriguing possibility that the promotion of endothelial regeneration may be a novel therapeutic strategy for ALI.
\end{abstract}

\section{Pathophysiology of acute lung injury}

Acute lung injury (ALI) and its more severe form, acute respiratory distress syndrome

Nonstandard abbreviations used: ALI, acute lung injury; ARDS, acute respiratory distress syndrome; FoxM1, forkhead box M1; FoxM1 CKO, endothelial cell-restricted FoxM1-deficient (mice).

Conflict of interest: The authors have declared that no conflict of interest exists.

Citation for this article: $J$. Clin. Invest. 116:2316-2319 (2006). doi:10.1172/JCI29637.
(ARDS), are characterized by an acute inflammatory process in the airspaces and lung parenchyma (1). These clinical syndromes are manifestations of the loss of barrier function of the alveolar epithelial and pulmonary capillary endothelial cells, resulting in respiratory failure. Epidemiological data suggest that the annual incidence of ALI/ARDS in the United States is 75 per 100,000 of the population. Although evidence exists that mortality in patients with ALI/ARDS has declined over the last 10 years, it remains high at $30 \%-40 \%$ and is still an important cause of death in critically ill patients.

The clinical course of ALI is a complex, variable process associated with severe lung dysfunction. The first stage, the exudative phase, is an acute inflammatory response accompanied by a marked influx of neutrophils injuring epithelial and endothelial cells (Figure 1). The resulting death of type I epithelial cells invites a breakdown in the gas exchange and barrier function of the lung and is associated with the flooding of airspaces with protein-rich edematous fluid. Histological features are dense hyaline membranes and alveolar collapse. Injury to type II epithelial cells reduces surfactant production and impairs the removal of edematous fluid from the alveolar space. Endothelial injury leads to a widening of 


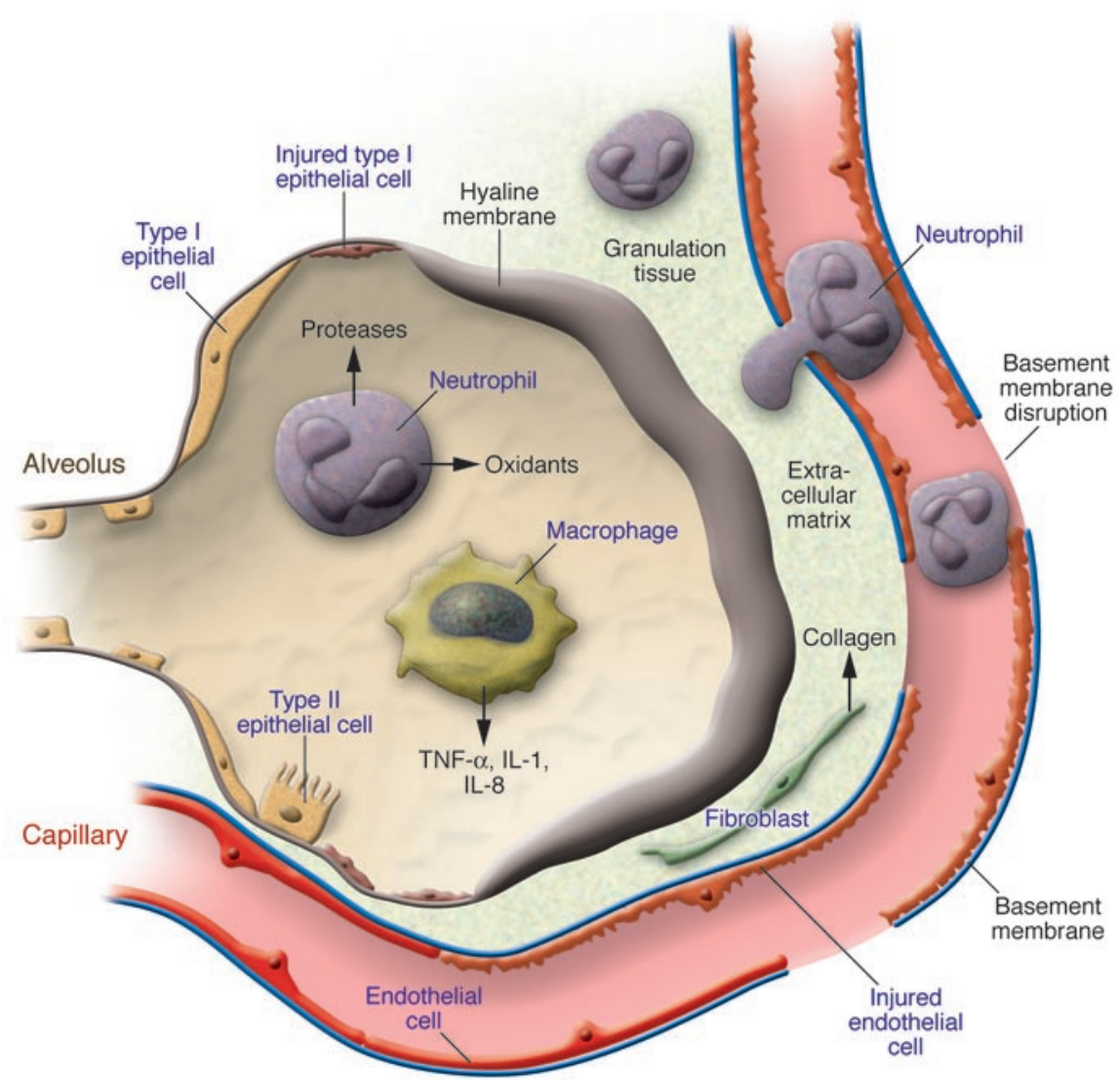

cellular junctions and capillary membrane disruption, producing capillary leakage and edema. The proliferative phase is characterized by type II cell proliferation with relining of the denuded basement membrane. This is accompanied by ingrowth of mesenchymal cells, such as fibroblasts, into granulation tissue, followed by the deposition of collagen and the migration of epithelial cells over the surface of organizing granulation tissue. Some patients have an uncomplicated course and the disorder rapidly resolves; however, insufficient repair leads to the fibrotic phase, which is identified by the deposition of excess collagen and extracellular matrices and is associated with alveolar wall fibrosis. A complex network of cytokines initiates and amplifies the inflammatory response in ALI. These molecules are produced locally in the lung by alveolar macrophages, epithelial cells, and fibroblasts and promote neutrophil-dependent injury to epithelial and endothelial cells. Imbalance between cell death by persistent injury and repair appears to be the main pathogenesis of ALI.

A recent prospective trial demonstrated that use of a ventilatory strategy with a low tidal volume significantly reduced in-hos- pital mortality (2), but clinical trials with other treatment strategies, such as surfactant-replacement therapy (3) and inhalation of nitric oxide, failed to show benefits (4). Pharmacological treatments with antiinflammatory agents, including glucocorticoids, have not proven beneficial (5), suggesting the complexity and redundancy of inflammation in ALI. Further understanding of the molecular mechanisms underlying ALI is needed.

\section{Emerging roles of the forkhead box M1 transcription factor in ALI}

The forkhead gene family, named after the founding gene member in Drosophila, is characterized by a unique DNA-binding domain (6). This so-called forkhead box encodes a winged-helix DNA-binding motif and is named after the structure of the domain when bound to DNA. The transcription factor forkhead box M1 (FoxM1) and other members of this family have been implicated in organogenesis during embryonic development and tumorigenesis in adulthood (7). Regulation of FoxM1 activity and its downstream target molecules is illustrated in Figure 2. FoxM1 is expressed during cellular proliferation and

\section{Figure 1}

Cellular mechanisms of ALI. Proinflammatory cytokines, such as TNF- $\alpha$, IL-1, and IL-8, are produced locally in the lung by alveolar macrophages, epithelial cells, and fibroblasts and promote neutrophil-dependent injury to epithelial and endothelial cells, which leads to the loss of barrier function of the alveolar epithelial and pulmonary capillary endothelial cells (basement membrane disruption). Insufficient repair leads to the deposition of excess collagen and extracellular matrices and is associated with alveolar wall fibrosis.

extinguished in terminally differentiated cells. FoxM1-deficient embryos die in utero due to severe defects in the development of the embryonic liver and heart (8). The FoxM1-deficient liver displays abnormal accumulation of polyploid hepatoblasts resulting from impaired DNA replication and mitosis. The FoxM1-deficient lung also displays severe abnormalities in the development of the pulmonary microvasculature that are associated with diminished expression of genes essential for lung morphogenesis, such as TGF- $\beta$ receptors and VEGF receptors (9). FoxM1 expression is increased during liver regeneration after injury. Premature expression of FoxM1 in the regenerating liver accelerates the onset of hepatocyte DNA replication and mitosis by modulating the expression of cell cycleregulator genes, such as cyclin-dependent kinase inhibitors, cyclins, and cell division cycle 25 (Cdc25) phosphatases toward proliferation (10). Conversely, hepatocyte-specific deletion of the FoxM1 gene markedly impairs liver regeneration after injury (11) and inhibits the development of hepatocellular carcinoma (12). The ubiquitous expression of FoxM1 accelerates the proliferation of distinct pulmonary cell types, such as epithelial and endothelial cells, after lung injury induced by chemicals (13), indicating that FoxM1 promotes proliferation of different types of cells.

In this issue of the JCI, Zhao et al. examine the potential role of FoxM1 in an LPSinduced ALI model using endothelial cell-restricted FoxM1-deficient (FoxM1 CKO) mice (14). In contrast to FoxM1null mice, approximately $80 \%$ of FoxM1 CKO mice developed normally and had a normal cardiovascular system. One possible explanation for this observation is that other members of the forkhead gene family may play a redundant role in cardiovascular development. Another possibility is that expression of FoxM1 in other 

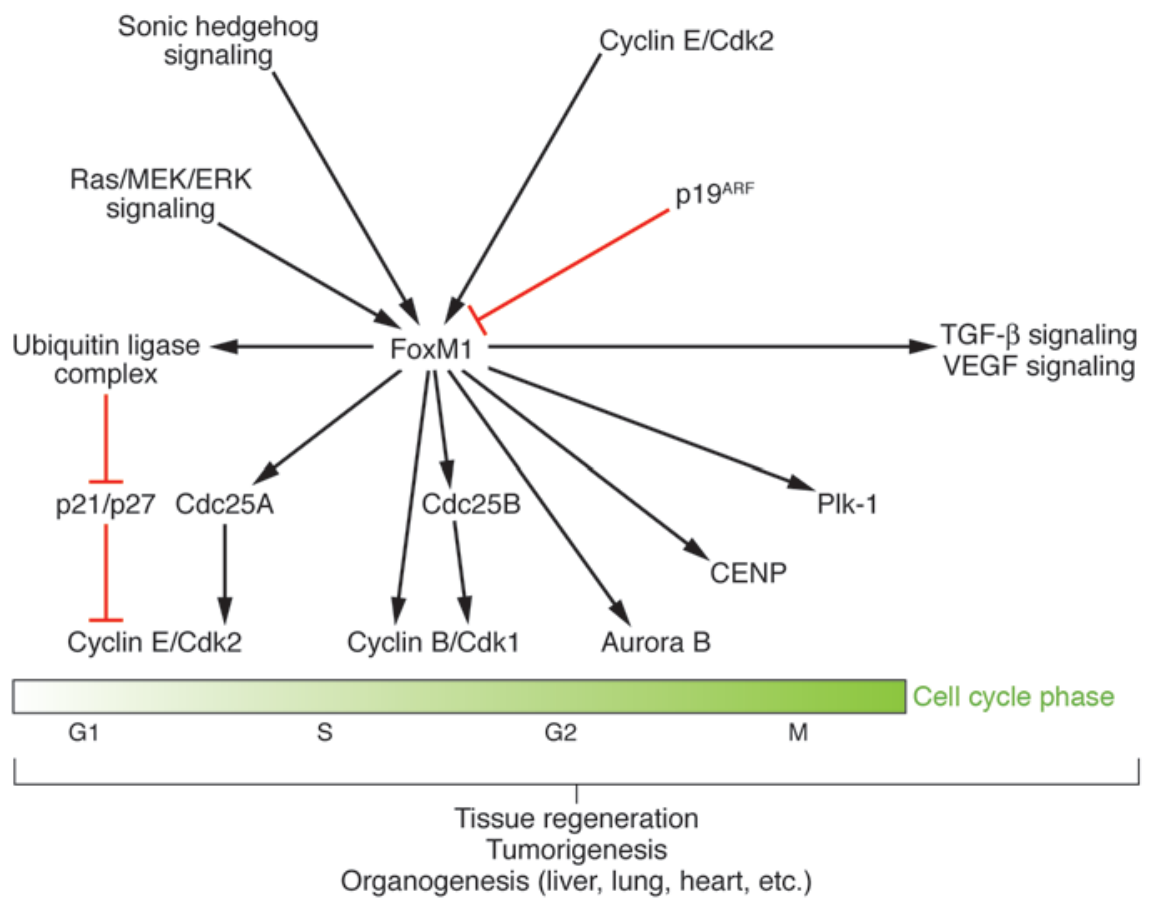

cell types may release factors that promote endothelial proliferation and survival, leading to normal cardiovascular development. In the present study, however, the reason that approximately $20 \%$ of FoxM1 $\mathrm{CKO}$ embryos died in utero remains to be determined. When ALI was induced by LPS, the pulmonary expression of FoxM1 was upregulated in wild-type mice. This upregulation was significantly reduced in the lungs of FoxM1 CKO mice. Until recently, only a few molecules that regulate FoxM1 activity have been identified (Figure 2). The authors found that 2 inflammatory mediators induced FoxM1 expression in vitro, but the mechanism of FoxM1 induction after LPS treatment has not been examined. They claim that endothelial cell-restricted deletion of FoxM1 markedly impaired pulmonary endothelial cell regeneration and increased vascular permeability, thus reducing survival after ALI due to severe pulmonary edema. Defective endothelial cell repair in FoxM1 CKO mice is not attributed to the deletion of FoxM1 in Tie2-positive bone marrow cells since transplantation of wild-type bone marrow cells into FoxM1 CKO mice had no effect on increased vascular permeability. Although deletion of FoxM1 might directly affect endothelial permeability, an initial increase in vascular permeability after LPS challenge as well as an increase in basal endothelial barrier function in the lung of FoxM1 CKO mice was similar to that of wild-type mice.
Whereas the extent of endothelial apoptosis after LPS treatment was not different between FoxM1 CKO and wild-type mice, endothelial proliferation was significantly impaired in the lungs of FoxM1 CKO animals after injury, suggesting that the lack of endothelial proliferation may be a major cause of the prolonged increase in vascular permeability. However, we cannot exclude the possibility that FoxM1 deficiency in other vascular beds might reduce the recovery of FoxM1 CKO mice after LPS challenge. It is interesting to note that recruitment of neutrophils and induction of pulmonary cytokine expression after lung injury occurred in FoxM1 CKO mice to a degree similar to that in wild-type mice, suggesting that the inflammatory response does not contribute to increased vascular permeability in this model. There was no difference in the number of apoptotic nonendothelial cells between FoxM1 CKO and wild-type mice after ALI, indicating that the extent of epithelial injury did not affect mortality following LPS-induced injury. It has been reported that LPS administration cannot completely mimic ALI induced by endotoxemia or bacteremia because epithelial injury is mild (15). Thus, it remains to be determined whether forced FoxM1 expression in the pulmonary endothelium reduces mortality in more severe ALI models. Restoring FoxM1 expression in the liver of aged mice was shown to improve age-associated decline in liver regeneration

\section{Figure 2}

Regulation of FoxM1 activity and its downstream target molecules. FoxM1 is positively regulated by sonic hedgehog, Ras/MEK/ERK, and cyclin E/Cdk2 growth signaling pathways, among others, and inhibited by the tumor suppressor p19ARF. FoxM1 directly or indirectly modulates a number of cell cycle (replication/mitosis) regulators to promote cell proliferation and affects the signal pathways essential for organogenesis, including TGF- $\beta$ signaling and VEGF signaling, thereby contributing to tissue regeneration, tumorigenesis, and organogenesis during embryonic development. Cdc25A, cell division cycle 25A; Cdc25B, cell division cycle 25B; CENP, centromere protein; Plk-1, polo-like kinase-1; p19ARF, 19-kDa alternative reading frame protein.

(16) whereas FoxM1 deficiency was reported to accelerate cellular aging (17), which has been implicated in age-related diseases, including human atherosclerosis (18). It would also be interesting to examine whether endothelial expression of FoxM1 prevents vascular aging and atherosclerosis. Finally, given the role of endothelial regeneration, FoxM1 is likely to contribute to neovascularization of ischemic tissues and may be a therapeutic target for ischemic cardiovascular diseases.

\section{Approach to ALI treatment}

From the insights provided in the present study (14), we now realize that endothelial regeneration is a potential strategy for ALI treatment. This may be accomplished by introducing FoxM1 or cell cycle-promoting genes into the pulmonary endothelium, although we must carefully examine its effects on tumorigenesis. Treatment with growth factors, such as hepatocyte growth factor and keratinocyte growth factor, has been reported to promote regeneration of epithelial and endothelial cells in animal models (19) and may be effective in patients with ALI. Another approach to ALI treatment could involve the use of stem cells to regenerate injured lung tissue. Bone marrow reconstitution studies have demonstrated that these stem cells have the potential to differentiate into epithelial and endothelial cells in the lung (20). Intravenous infusion of 
bone marrow-derived stem cells may be beneficial in ALI since pulmonary microvasculature would be the first capillary bed encountered and thus entrap stem cells that may help to regenerate pulmonary endothelium after injury. Although considerable work will be required, promotion of endothelial regeneration would be a novel approach to treat ALI.

Address correspondence to: Issei Komuro, Department of Cardiovascular Science and Medicine, Chiba University Graduate School of Medicine, 1-8-1 Inohana, Chuoku, Chiba 260-8670, Japan. Phone: 81-43226-2097; Fax: 81-43-226-2557; E-mail: komuro-tky@umin.ac.jp.

1. Ware, L.B., and Matthay, M.A. 2000. The acute respiratory distress syndrome. N. Engl. J. Med. 342:1334-1349.

2. [Anonymous]. 2000. Ventilation with lower tidal volumes as compared with traditional tidal volumes for acute lung injury and the acute respiratory distress syndrome. The Acute Respiratory Distress Syndrome Network. N. Engl. J. Med. 342:1301-1308.

3. Anzueto, A., et al. 1996. Aerosolized surfactant in adults with sepsis-induced acute respiratory distress syndrome. Exosurf Acute Respiratory Distress Syndrome Sepsis Study Group. N. Engl.J. Med.
334:1417-1421.

4. Dellinger, R.P., et al. 1998. Effects of inhaled nitric oxide in patients with acute respiratory distress syndrome: results of a randomized phase II trial. Inhaled Nitric Oxide in ARDS Study Group. Crit. Care Med. 26:15-23.

5. Bernard, G.R., et al. 1987. High-dose corticosteroids in patients with the adult respiratory distress syndrome. N. Engl. J. Med. 317:1565-1570.

6. Kaestner, K.H., Knochel, W., and Martinez, D.E. 2000 Unified nomenclature for the winged helix/forkhead transcription factors. Genes Dev. 14:142-146.

7. Costa, R.H., Kalinichenko, V.V., Major, M.L., and Raychaudhuri, P. 2005. New and unexpected: forkhead meets ARF. Curr. Opin. Genet. Dev. 15:42-48.

8. Korver, W., et al. 1998. Uncoupling of S phase and mitosis in cardiomyocytes and hepatocytes lacking the winged-helix transcription factor Trident. Curr. Biol. 8:1327-1330.

9. Kim, I.M., et al. 2005. The forkhead box $\mathrm{m} 1 \mathrm{tran}-$ scription factor is essential for embryonic development of pulmonary vasculature. J. Biol. Chem. 280:22278-22286.

10. Ye, H., Holterman, A.X., Yoo, K.W., Franks, R.R., and Costa, R.H. 1999. Premature expression of the winged helix transcription factor HFH-11B in regenerating mouse liver accelerates hepatocyte entry into S phase. Mol. Cell. Biol. 19:8570-8580.

11. Wang, X., Kiyokawa, H., Dennewitz, M.B., and Costa, R.H. 2002. The Forkhead Box m1b transcription factor is essential for hepatocyte DNA replication and mitosis during mouse liver regeneration. Proc. Natl. Acad. Sci. U. S. A. 99:16881-16886.

12. Kalinichenko, V.V., et al. 2004. Foxm1b transcription factor is essential for development of hepa- tocellular carcinomas and is negatively regulated by the p19ARF tumor suppressor. Genes Dev. 18:830-850.

13. Kalinichenko, V.V., et al. 2003. Ubiquitous expression of the forkhead box M1B transgene accelerates proliferation of distinct pulmonary cell types following lung injury. J. Biol. Chem. 278:37888-37894.

14. Zhao, Y.-Y., et al. 2006. Endothelial cell-restricted disruption of FoxM1 impairs endothelial repair following LPS-induced vascular injury. J. Clin. Invest. 116:2333-2343. doi:10.1172/JCI27154.

15. Wiener-Kronish, J.P., Albertine, K.H., and Matthay, M.A. 1991. Differential responses of the endothelial and epithelial barriers of the lung in sheep to Escherichia coli endotoxin. J. Clin. Invest. 88:864-875.

16. Wang, X., et al. 2001. Increased levels of forkhead box M1B transcription factor in transgenic mouse hepatocytes prevent age-related proliferation defects in regenerating liver. Proc. Natl. Acad. Sci. U. S. A. 98:11468-11473.

17. Wang, I.C., et al. 2005. Forkhead box M1 regulates the transcriptional network of genes essential for mitotic progression and genes encoding the SCF (Skp2-Cks1) ubiquitin ligase. Mol. Cell. Biol. 25:10875-10894.

18. Minamino, T., et al. 2004. Vascular cell senescence and vascular aging. J. Mol. Cell. Cardiol. 36:175-183.

19. Ware, L.B., and Matthay, M.A. 2002. Keratinocyte and hepatocyte growth factors in the lung: roles in lung development, inflammation, and repair. Am. J. Physiol. Lung Cell. Mol. Physiol. 282:L924-L940.

20. Krause, D.S., et al. 2001. Multi-organ, multi-lineage engraftment by a single bone marrow-derived stem cell. Cell. 105:369-377.

\section{Toll-like receptors and IFN- $\alpha$ : partners in autoimmunity}

Marco Colonna

Department of Pathology and Immunology, Washington University School of Medicine, St. Louis, Missouri, USA.

\begin{abstract}
Many autoimmune diseases are thought to be precipitated by viral infections. In this issue of the JCI, Lang et al. demonstrate that, in a mouse model of autoimmune hepatitis, viral infections not only trigger expansion of self-reactive $T$ cells but also activate antigen-presenting cells through TLR stimulation (see the related article beginning on page 2456). Activated cells then secrete IFN- $\alpha$ and TNF- $\alpha$, which trigger tissue release of chemokines that attract self-reactive $\mathrm{CD8}^{+} \mathrm{T}$ cells, ultimately leading to liver damage.
\end{abstract}

Autoimmune diseases result from the propagation of $\mathrm{T}$ and $\mathrm{B}$ cells that recognize self antigens and mediate tissue destruction.

Nonstandard abbreviations used: CXCL9, CXC chemokine ligand 9; GP, glycoprotein 1 -60; gp33, LCMV-glycoprotein peptide $33-41$; IFNAR, IFN receptor $\alpha$; LCMV, lymphocytic choriomeningitis virus; PKR, doublestranded RNA-dependent protein kinase; poly(I:C), polyinosinic-polycytidylic acid.

Conflict of interest: The author has declared that no conflict of interest exists.

Citation for this article: J. Clin. Invest. 116:2319-2322 (2006). doi:10.1172/JCI29879.
Normally, self-reactive lymphocytes are deleted in central lymphoid organs, the thymus and bone marrow, during development (1). In the periphery, multiple safeguards exist to further prevent activation of selfreactive lymphocytes that have eluded central elimination (2). Thus, autoimmunity is fundamentally due to failure of central and/ or peripheral mechanisms of immunological tolerance. Viral infections have long been suspected to instigate or overtly precipitate autoimmunity (3-5). Viral antigens can trigger autoimmune responses by molecular mimicry of self structures. Virus-mediated tissue destruction may also generate novel tissue-specific antigens to which $\mathrm{T}$ cells are not tolerant. Moreover, antiviral immune responses can trigger the release of cytokines that induce bystander activation of autoreactive $T$ cells. In this issue of the JCI, Lang et al. demonstrate that viruses can initiate autoimmune damage through yet another mechanism (6). In a mouse model of hepatitis, lymphocytic choriomeningitis virus (LCMV) induced IFN- $\alpha$ secretion through TLR3. In turn, IFN- $\alpha$ triggered secretion of chemokines that attract autoreactive $T$ cells into the liver, thereby causing hepatitis.

To model liver-specific expression of a self-antigen, Lang et al. (6) used transgenic mice that express the LCMV-glycoprotein ${ }_{1-60}$ (LCMV-GP) under the control of the mouse albumin promoter (Alb-1 mice). The LCMVglycoprotein peptide $33-41$ (gp33) is presented 